\title{
New approaches in mineral raw materials comminution tests modelling
}

\author{
L.S. Chitalov \\ Postgraduate student, St. Petersburg mining University, St. Petersburg, Russia \\ V.V. Lvov \\ PhD, Associate professor, St. Petersburg mining University, St. Petersburg, Russia
}

\begin{abstract}
The paper discusses a model of ore particles crushing with a JK Drop Weight Tester, created using the method of discrete elements in the Rocky DEM software. The mathematical relationship between output parameters of the JK Drop Weight Test (JK DWT) and parameters of the breakage model in Rocky DEM is shown. A JK DWT was performed on a technological sample of copper-nickel ore. The JK DWT results were used in Rocky DEM to calibrate its breakage model by factorial experiment using two parameters that are not related to the results of the JK DWT. The achieved accuracy of the resulting model is $\pm 3.1 \%$ of the $t_{10}$ value. The resulting model can be used to obtain the particle size distribution of crushing products for various comminution machines, as well as for determination the parameters for calibrating mathematical models of crushers, autogenous and semi-autogenous mills in the JKSimMet.
\end{abstract}

\section{INTRODUCTION}

In the practice of mineral processing, comminution processes are the most expensive due to their high energy consumption. Today, the main way of reducing energy costs for crushing and grinding is optimizing these processes through mathematical modeling. At the same time, no modeling can be carried out without the strength characteristics of mineral raw materials (Gospodarikov \& Zatsepin, 2019, Zuev et al., 2019). Obtaining such characteristics is closely related to laboratory tests, which describe the relationship between the change in the size of the material and the energy expended on this change. The JK Drop Weight Test is one of the most used worldwide comminution test. Its results may be used as inputs parameters for calibration different kind of breakage models. One of them, constructed with the discrete element method, was calibrated in this study (Taranov et al., 2013).

\section{$1.1 \quad J K$ drop weight test}

JK Drop Weight Test (JK DWT) (Nikolaeva et al., 2017, Andreev et al., 2012) of JKTech (Australia) is developed by the Julius Kruttschnitt Mineral Research Centre. Power measurements in the range of size (from 13 to $63 \mathrm{~mm}$ ) at different energy levels (from 0.1 to $2.5 \mathrm{kWh} /$ t) were obtained in the test for copper-nickel ore. The equipment for determining the breakage function is called the JK Drop Weight Tester (JK DW). Test results were reduced to three parameters - $A, b$ and $t_{a}$ for subsequent use in the JKSimMet software.

\section{$1.2 \mathrm{JKSimMet}$}

When modeling in JKSimMet, the ore parameters are combined with the comminution equipment details and operating conditions, which allow to predict the performance of autogenous 
grinding and semi-autogenous grinding mills (AG/SAG), crushers and other comminution equipment, as well as to obtain information on the particle size distribution. Using JKSimMet in conjunctions with the JKSimFloat software it is possible to optimize existing or design new flowsheets for comminution circuits and flowsheets that include flotation operations (Alexandrova et al., 2019).

Despite the fact that JKSimMet is a leader in its field, its computational system is fixed; the simulation itself is static and does not take into account all the factors that affect the process. So, as the parts of the ore dressing machine wear out, there are modifications in the technological processes occurring. Such changes are not taken into account when modeling in JKSimMet.

\subsection{Discrete Element Method (DEM)}

The discrete element method as applied in mining is a method of modeling physical processes in which step-by-step calculation of particles kinetics of their motions and collisions with other particles and machine construction is carried out for individual particles. DEM simulations are widely used for AG/SAG processes, but as a rule only with a qualitative assessment of the dynamics of mill load (Cleary et al., 2003, Weerasrkara et al., 2013). Recently, the Rocky DEM software (Rocky) has the ability to model particle breakage processes (Beloglazov, 2018), in which the parameters obtained by JK DWT are basic. However, due to a number of limitations of DEM modeling, direct input of JK DWT results into the Rocky interface is not possible. At the same time, Rocky's mathematical model is easy to control, and the modeling process is dynamic.

\section{MATERIALS AND METHODS}

\subsection{The subject, tasks and the aim of the study}

The aim of this work is to combine the mathematical aspects of the breakage model of mineral raw materials in Rocky DEM software in line with the results of the drop weight test for the material (model verification). The object of the study was a copper-nickel ore sample.

The tasks include carrying out laboratory tests according to the JK DWT method on a technological sample of copper-nickel ore; comparing the theoretical foundations of JK DWT and the mathematical apparatus of breakage model in Rocky; verification of mineral breakage modeling by the JK DWT using the discrete element method in Rocky DEM software by means of a complete model factor experiment.

Subsequently, a calibrated fracture model in Rocky can be used to obtain data that allows the calibration of mathematical models of hand-held apparatuses in JKSimMet, as well as to clarify the effect of abrasive wear of the apparatuses construction on the course of the technological process.

\subsection{Methods}

The output parameters of the JK DWT are $A$ and $b$ characterizing the strength properties of the tested ore. These constants are found after obtaining all the results of sieve analyses of the fracture products and bringing them to the exponential dependence of the following form (see Figure 1):

$$
t_{10}=A \cdot\left(1-\exp ^{-b \cdot E_{c s}}\right)
$$

where $t_{10}$ is the percentage finer than $1 / 10$ th of the original particle size $\% ; A$ - constant (asymptotic, Figure 1); $b$ - constant (controls the shape of the curve); $E_{c s}$ - specific energy, $\mathrm{kWh} / \mathrm{t}$, in JK DWT $E_{c s}$ is regulated by the mass of the drop weight and the drop height (equation 2). 


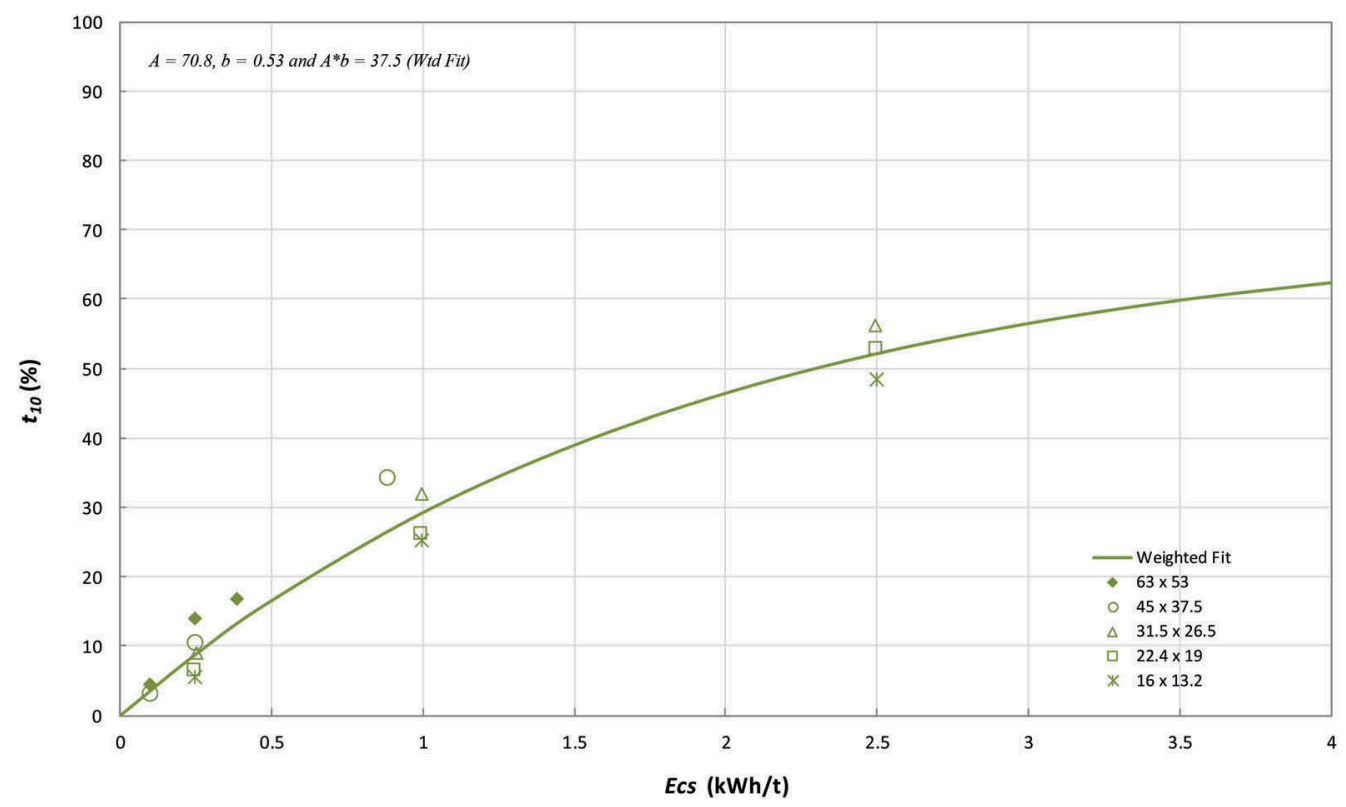

Figure 1. The dependence of the parameter $t_{10}$ on the specific energy Ecs obtained from the JK DWT results for the sample of copper-nickel ore.

$$
E_{c s}=\frac{m g h}{3,6 m_{y}}
$$

where $m$ is the mass of the weight, $\mathrm{kg} ; m_{u}$ - particle mass, $\mathrm{g} ; g$ is the force due to gravity, $m / s^{2} ; h$ - "clean" drop height (difference between final $h_{2}$ and initial $h_{1}$ position of the steel weight, $h_{2}$ varies as it depends on the residue after breakage.), $\mathrm{m}$.

In Rocky, the model multi-step events of destruction is transformed considering the probability of an act of particle breaking:

$$
P\left(e_{\text {cum }}\right)=1-\exp ^{- \text {SecumL } / \text { Lref }},
$$

where $P\left(e_{\text {cum }}\right)$ - probability of breakage at the current value of the total specific energy applied to the particle $\left(e_{\text {cum }}\right), S$ - constant for the material, called the coefficient of the selection function or the selective coefficient, $\mathrm{kg} / \mathrm{J} ; L$ - particle size, $\mathrm{m} ; L_{r e f}$-reference particle size, $\mathrm{m}$.

In this case, $e_{c u m}$ is the sum of the energies applied to the particle in the current and previous calculation steps $t$ :

$$
e_{c u m}^{t}=e_{c u m}^{t-\Delta t}+\left(e_{c}^{t}-e_{c}^{t-\Delta t}\right)
$$

where $e_{c u m}^{t-\Delta t}$ and $e_{c}^{t-\Delta t}$ - total specific contact energy and instantaneous specific contact energy at the previous time step $t$, respectively.

In addition, in Rocky, for the act of breakage to occur, the specific energy applied to the particle must be greater than the minimum specific energy of destruction, $e_{\text {min }}$, which is determined by the expression:

$$
e_{\min }=e_{\min \cdot r e f} \frac{L_{r e f}}{L}
$$


where $e_{\text {min.ref }}$ - reference minimum specific energy for the reference particle size of this material. This parameter, among others, is introduced into the fracture model when it is configured in the Rocky interface.

When a particle breaks, fragments are generated in accordance with the Voronoi breakage algorithm (Aurenhammer \& Klein, 2000) in accordance with the minimum size of the generated particles, which is set by the user. The value of the parameter $t_{10}$, which will eventually be generated by the model, is calculated by the expression:

$$
t_{10}=A\left[1-\exp \left(-S e_{c u m} L / L_{r e f}\right)\right]
$$

The expression $\left(e_{c u m} L / L_{r e f}\right)$ in equation 5 corresponds to the $E_{c s}$ from equation 1 taking into account the size of a particular destructible particle. Thus, to obtain a similar final fracture result in the Rocky JK DW model (see Figure 2, a), the parameters of the minimum specific energy $e_{\min }$ from equation 4 and the selective fracture function $S$ from equations 2 and 5 were calibrated.

$$
b=\frac{S L e_{c u m}}{L_{r e f} E_{c s}}=\frac{S e_{c u m} e_{\min . r e f}}{E_{c s} e_{\min }}
$$

To solve this problem, a complete factorial model experiment was conducted on the values of the coefficients $S$ and $e_{\text {min }}$ comparing the parameter $t_{10}$ obtained on their basis with the results of JK DWT carried out on a sample of the studied copper-nickel ore. To carry out a factor experiment, ANSYS Workbench software was performed, which partially automates the input parameters to the Rocky JK DW model, among which were:

- The mass of the drop weight (8 values for 15 experiments),

- Drop height (15 values for 15 experiments),

- The particle size (5 values for 15 experiments), it was also used as $L_{r e f}$,

- Particle mass (15 values for 15 experiments),

Among the varied parameters of the fracture model for each of the 15 experiments were:

- The reference minimum value of specific energy $e_{\text {min }}$ from equation 4 (7 values),

- Selective coefficient $S$ from equations 2 and 5 (7 values).

a)
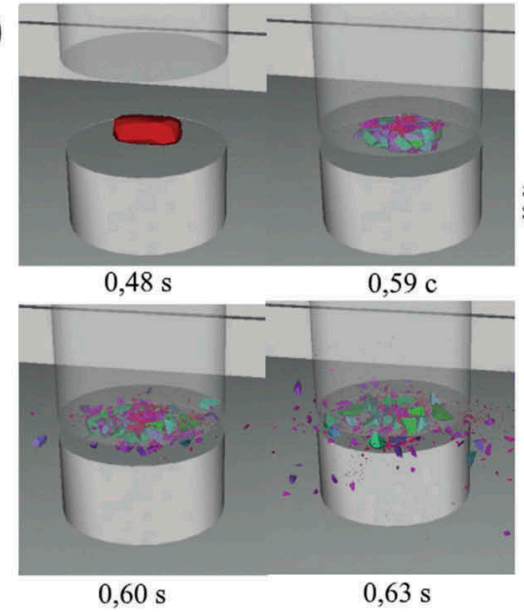

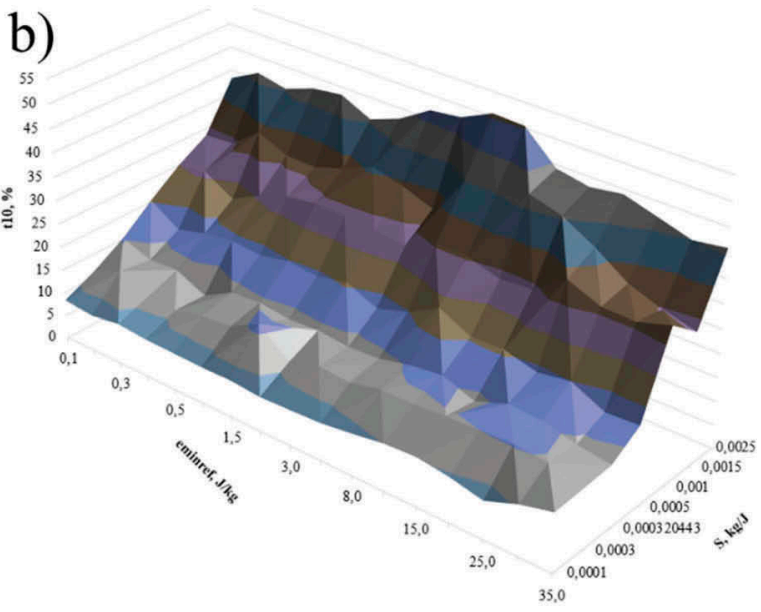

Figure 2. JK DWT modeling: a) a tester in the form of a 3D model; b) a surface diagram for the values of the obtained parameter $t_{10}$ when varying the studied factors. 
The constant $A$ form equation 5 was introduced directly into the Rocky model from the results of a JK DWT. The size of the smallest generated particles in each experiment was introduced as $1 / 50$ of the arithmetic mean particle size of the destructible particle size class.

\section{RESULTS AND DISCUSSION}

A series of experiments was simulated with 49 models for each of 15 sets of JK DWT (735 models in total). The output was reduced to surface diagrams of parameter $t_{10}$ for each of the 15 sets (one of such diagrams is shown in Figure 2, b).

It was established that the influence of the input reference minimum specific energy emin. ref on the value of the output parameter $t_{10}$ is insignificant in comparison with the influence of the selective coefficient $\mathrm{S}$ (see Figure 2, b). This is explained by the specificity of the destruction of the material using the JK DW installation, aimed at creating a single act of destruction by each of the particles included in the set.

The results obtained for equation 7 are presented in Table 1.

Figure 3 shows the initial dependence $t_{10}=f(A, b)$ and the same dependence obtained from the DEM simulation of the JK DW installation in Rocky.

Table 2 presents the initial strength parameters $A$ and $b$ obtained from Rocky models.

Table 1. The absolute error in determining the value $t_{10}, \%$.

\begin{tabular}{llllll}
\hline Specific energy, kWh/t & Sizes, $\mathrm{mm}$ & & & \\
\cline { 2 - 6 } & $-63+53$ & $-45+37,5$ & $-31,5+26,5$ & $-22,4+19$ & $-16+13,2$ \\
\hline 0,10 & $-0,31$ & 0,13 & - & - & - \\
0,25 & 1,17 & 1,01 & $-1,20$ & 0,01 & 0,15 \\
0,40 & 0,13 & - & - & - & - \\
1,00 & - & $-2,70$ & 2,12 & $-0,90$ & $-1,43$ \\
2,50 & - & - & 3,07 & $-3,06$ & 1,81 \\
\hline
\end{tabular}

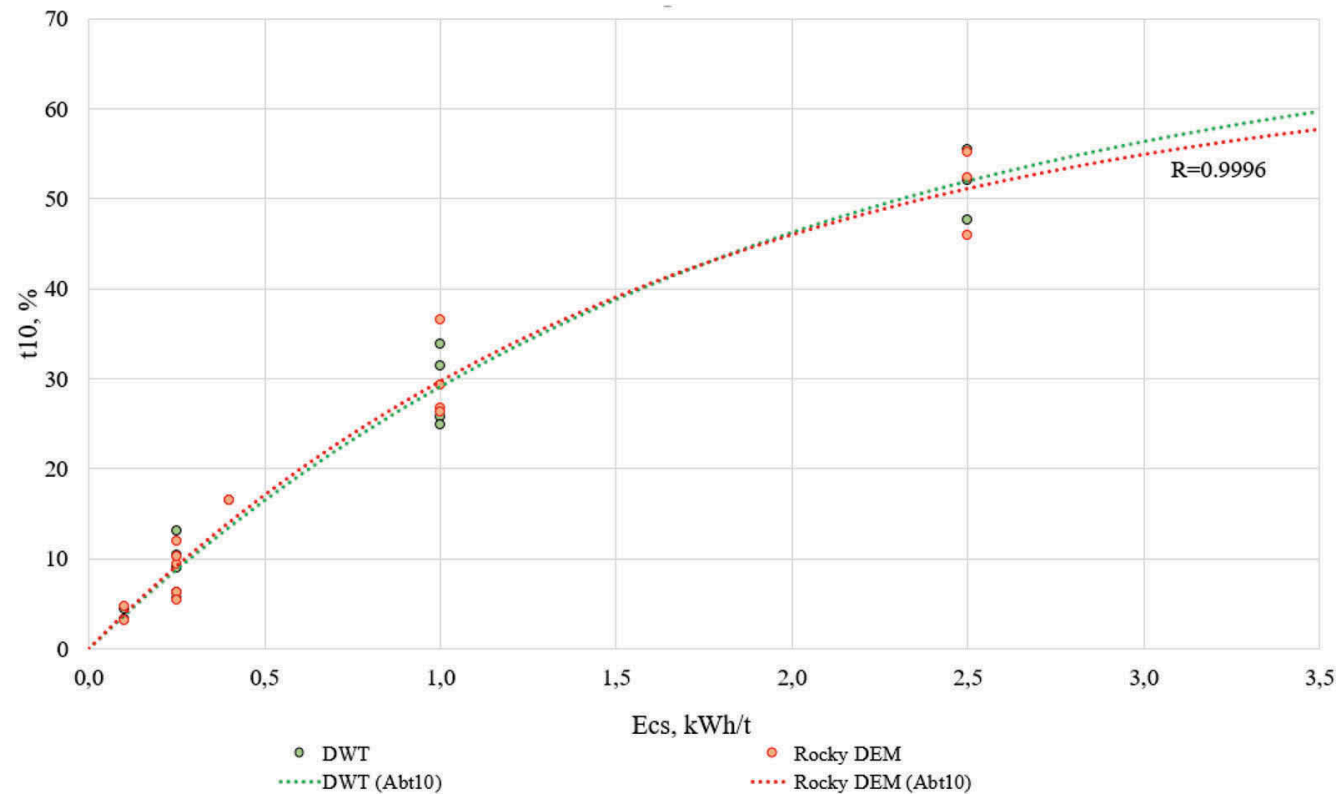

Figure 3. The dependence obtained from the results of DEM modelling. 
Table 2. Strength parameters obtained by the

DEM model.

\begin{tabular}{lcc}
\hline Parameter & JK DWT & Rocky DEM \\
\hline $\mathrm{A}$ & 70,8 & 65,7 \\
$\mathrm{~b}$ & 0,53 & 0,60 \\
$\mathrm{Ab}$ & 37,5 & 39,6 \\
\hline
\end{tabular}

\section{CONCLUSIONS}

According to the results of the work, the coefficients of the mathematical model in Rocky, making it possible to predict the parameter t10 obtained in the JK DWT experiments for various values of the specific breakage energy for all studied particle size fractions with an accuracy of $\pm 3.1 \%$ at a confidence interval of $95 \%$. The repeatability of the model has not been estimated with many tests. Despite this, the resulting model allows to calibrate mathematical models in JKSimMet software for such ore dressing equipment as crushers and AG/SAG mills according to the power parameters developed under load and particle size distribution of the feed and crushing product taking into account the lining wear rate.

The calibrated breakage Rocky DEM model may be used for analysis of particle size distribution changes during dry crushing and grinding processes of the studied nick-el-copper ore sample and during JKSimMet equipment settings:

- When making structural changes to ore preparation machines (using non-standard discharge gratings in AG mills, using non-standard lining in crushers, etc.);

- With the abrasive wear of the constructions of comminution equipment;

- To analyze the equipment which is not included in the list of supported in JKSimMet software.

- The research was carried out under the grant received from Russian Foundation of Fundamental Research № 20-55-12002

\section{REFERENCES}

Aleksandrova T. N., Nikolaeva N.V., Lvov V.V., Romashev A.O. 2019. Ore processing efficiency improvements for precious metals based on process simulations. Obogashchenie rud, No. 2: 8-13. DOI: 10.17580 /or.2019.02.02.

Andreev Ye.Ye., Zakhvatkin V.V., Lvov V.V., Nikolaeva N.V. 2019. Application of falling weight test for estimation of ore strength properties. Obogashchenie rud, No. 3: 19-22.

Aurenhammer F., Klein R. 2000. Voronoi Diagrams. Handbook of Computational Geometryю In J. R. Sack and J. Urrutia (ed.) Amsterdam, Holland: North-Holland: 201-290.

Beloglazov I.I., Stepanyan A.S., Feoktistov A.Yu., Yusupov G.A. 2018. Disintegration process modeling for a jaw crusher with complex jaws swing. Obogashchenie rud, No. 2: 3-8. DOI: 10.17580/ or.2018.02.01.

Cleary P.W., Morrisson R., Morrell S. 2003. Comparison of DEM and experiment for a scale model SAG mill. Mineral Processing, No. 68: 129-165.

Gospodarikov A.P. \& Zatsepin, M.A. 2019. Mathematical modeling of boundary problems in geomechanics. Gornyi Zhurnal, Issue 12: 16-20.

Nikolaeva N.V., Aleksandrova, T.N., Taranov, V.A. 2017. Determination of the degree of impact destruction of gold-bearing ore particles in the layer. Information (Japan), 20(9): 6605-6613.

Taranov, V.A., Baranov V.F., Aleksandrova T.N. 2013. Review of software for modeling and calculation of ore preparation flowsheets. Obogashchenie rud, No. 5: 3-7.

Weerasekara N.S., Powell M.S., Cleary P.W., Tavares L.M., Evertsson M., Morrison R.D., Quist J., Carvalho R.M. 2013. The contribution of DEM to the science of comminution. Powder Technology no. 248: 3-24.

Zuev B.Y., Zubov V.P., Smychnik A.D. 2019. Determination of static and dynamic stresses in physical models of layered and block rock masses. Gornyi Zhurnal, Issue 7: 61-66. 